\title{
Characteristic Analysis of Response Threshold Model and Its Application for Self-organizing Network Control
}

\author{
Takuya Iwai, Naoki Wakamiya, and Masayuki Murata \\ Graduate School of Information Science and Technology, \\ Osaka University, Suita, Osaka 565-0871, Japan \\ $\{$ t-iwai, wakamiya, murata\}@ist.osaka-u.ac.jp
}

\begin{abstract}
There is an emerging research area to adopt bio-inspired algorithms to self-organize an information network system. Despite strong interests on their benefits, i.e. high robustness, adaptability, and scalability, the behavior of bio-inspired algorithms under non-negligible perturbation such as loss of information and failure of nodes observed in the realistic environment is not well investigated. Because of lack of knowledge, none can clearly identify the range of application of a bio-inspired algorithm to challenging issues of information networks. Therefore, to tackle the problem and accelerate researches in this area, we need to understand characteristics of bio-inspired algorithms from the perspective of network control. In this paper, taking a response threshold model as an example, we discuss the robustness and adaptability of bio-inspired model and its application to network control. Through simulation experiments and mathematical analysis, we show an existence condition of the equilibrium state in the lossy environment. We also clarify the influence of the environmental condition and control parameters on the transient behavior and the recovery time.
\end{abstract}

Keywords: self-organization, response threshold model, robustness, adaptability, linear stability theory.

\section{Introduction}

For information networks to remain one of infrastructures indispensable for a safe, secure, and comfortable society, they must be more robust, adaptive, and scalable against ever-increasing size, complexity, and dynamics. To this end, researchers focus on self-organizing behavior of biological systems, where a global pattern emerges from mutual and local interactions among simple individuals, and develop novel control mechanisms [1].

Bio-inspired control mechanisms not only mimic behavior of biological organisms but are based on nonlinear mathematical models which explain or reproduce biological self-organization. Since bio-inspired mathematical models, which we call bio-models in this paper, are shown to have excellent characteristics, e.g. high convergence and stability, network control mechanisms based on bio-models 
are expected to be robust, adaptive, and scalable [2 -5]. Successful attempts published in literatures support this expectation and there is no doubt about the usefulness of bio-models 6,7 ].

However, bio-models are not necessarily versatile. One can achieve the best performance in one environment while it is useless in other. Furthermore, a bioinspired network control mechanism often experiences a variety of perturbation such as loss of information and failure of node and as a result it would fail in providing intended results in the actual environment. Therefore, we need deep understanding of bio-models especially in regard to their fundamental limits and applicability to network control suffering from perturbations. For example, they evaluated the influence of delay on a bio-inspired synchronization mechanism adopting the pulse-coupled oscillator model and showed that the synchronization error became as much as the propagation delay at the worst cases [8]. In [9], it is shown that the maximum rate of information loss that autonomous pattern formation based on the reaction diffusion model of biological morphogenesis can tolerate was as high as $35 \%$.

In this paper, by taking a response threshold model [10] as an example, we analyze the influence of information loss and node failure on the stability of a response threshold model-based network control. The response threshold model is a mathematical model of division of labors in a colony of social insects. It has been applied to variety of self-organizing network control, such as task allocation [11], topology control [12], routing [13] and cache replacement [14]. First, we evaluate the influence of information loss on autonomous task allocation. Simulation results show that the number of workers increases as the loss rate is higher while the loss rate does not have serious impact on the recovery time from node failure. Next, to clarify the maximum loss rate that response threshold model-based task allocation can tolerate, we conduct mathematical analysis of the model in lossy environment and derive existence conditions of an equilibrium state. Then, we formulate the recovery time and clarify important parameters to shorten it.

The remainder of this paper is organized as follows. First, in section 2 we briefly introduce a response threshold model. Next, in section 3, we evaluate the influence of information loss on transient behavior during recovery from node failures through simulation experiments. Then, in section 4 we build an analytical model and investigate the relationship between parameters and transient behavior. Finally, in section [5] we conclude this paper.

\section{Mathematical Model of Division of Labors}

A response threshold model [10] is a mathematical model which imitates a mechanism of adaptive division of labors in a colony of social insects. A colony is divided into two groups of workers and non-workers based on autonomous decision of individuals. The size of each group is well adjusted to meet the task-associated demand or stimulus intensity. In the following, we consider there is one task to be performed in the colony for the sake of simplicity of explanation. 
Let $s(k)(\geq 0)$ be the task-associated stimulus intensity at discrete time step $k$. The stimulus intensity gradually increases over time, and it decreases as individuals work. Its dynamics is formulated by the following discrete equation.

$$
s(k+1)=s(k)+\delta-\frac{w(k)}{M}
$$

Here constant $\delta(0 \leq \delta \leq 1)$ is the increasing rate of the stimulus intensity. $w(k)$ is the number of workers at time $k . M(>0)$ is the total number of individuals which are capable of performing the task. Based on the model, the stimulus intensity becomes stable when the ratio of workers in the colony is equal to $\delta$. The model can easily be extended to consider the absolute number of workers not the ratio by appropriately defining $\delta$.

By being simulated by the stimulus, each individual stochastically decides whether to perform the task. The state of individual $i$ at time $k$ is denoted as $X_{i}(k) \in\{0,1\}$, where 0 means it is a non-worker and 1 does a worker. The probability $P\left(X_{i}(k)=0 \rightarrow X_{i}(k+1)=1\right)$ that non-worker $i$ becomes a worker and begins performing the task at time $k+1$ is given by the following equation.

$$
P\left(X_{i}(k)=0 \rightarrow X_{i}(k+1)=1\right)=\frac{s^{2}(k)}{s^{2}(k)+\theta_{i}^{2}(k)}
$$

Here $\theta_{i}(k)(>0)$ is a threshold at time $k$, which corresponds to hesitation of individual $i$ in performing the task. Therefore, an individual with a smaller threshold is more likely to become a worker more often than those with a larger threshold.

The probability $P\left(X_{i}(k)=1 \rightarrow X_{i}(k+1)=0\right)$ that individual $i$, a worker, quits working at time $k+1$ is given by constant $p(0 \leq p \leq 1)$.

$$
P\left(X_{i}(k)=1 \rightarrow X_{i}(k+1)=0\right)=p
$$

Quitting a task at the constant rate enables rotation of the task among individuals, that is, work-sharing or load balancing. Given $p$, the average duration that an individual performs the task becomes $1 / p$.

When the number of idle individuals occasionally increases by addition of newcomers or the number of workers decreases for sudden death, the stimulus intensity eventually increases and individuals with high threshold turn into workers. Consequently, the ratio of workers is maintained at around the equilibrium point determined by the increasing rate $\delta$.

\section{Simulation-Based Analysis of Response Threshold Model}

Through simulation experiments, we evaluate transient behavior during recovery from death of individuals or node failures. 
Table 1. Parameter setting

\begin{tabular}{lll} 
Notation & Description & Default \\
\hline$\theta$ & Hesitation to become a worker & 10 \\
$p$ & Probability of quitting & 0.1 \\
$I_{c}$ & Interval between two successive stimulus diffusion & 1 \\
$I_{d}$ & Interval between two successive data reporting & 1 \\
$\delta$ & Increasing rate of stimulus intensity & 0.15 \\
$M$ & Total number of nodes & 1,000 \\
\hline
\end{tabular}

\subsection{Simulation Model}

We assume a hypothetical system consisting of a central unit and nodes among which a task is assigned. Nodes are homogeneous and capable of performing the task. The stimulus intensity is diffused to nodes from a central unit. Then as a response to the stimulus each node which received the stimulus decides whether to perform the task or not and reports the decision to the central unit. Those nodes that cannot receive the stimulus do not change their state. The central unit then obtains the number of workers $w(k)$ from received reports and derives the new stimulus intensity by using Eq. (1). The stimulus intensity is again diffused to nodes. While doing a task, a node would inform a central unit of its result. For example, in a case of a monitoring application of a wireless sensor network, each sensor node periodically sends a message containing sensor reading, such as temperature and humidity, to a sink. To take into account a realistic application of the response threshold model, we further assume that exchanges of the stimulus and responses is performed per $I_{c}(>0)$ second, corresponding to the control interval of an application system. Furthermore, the data reporting interval is assumed to be $I_{d}\left(0<I_{d} \leq I_{c}\right)$ second.

In the above mentioned system, there are three occasions for disruption of communication between a central unit and a node. A central unit sometimes fails in receiving a response from a node, which we call worker information, for communication errors. As a result, the stimulus intensity will be derived from the wrong number of workers. A central unit would also fail in receiving data from nodes. On the contrary, a node would fail in receiving what we call stimulus information from the central unit and cannot decide its new state. In the analysis, we denote the probability of loss of information as $q_{w}\left(0 \leq q_{w} \leq 1\right)$, $q_{d}\left(0 \leq q_{d} \leq 1\right)$ and $q_{s}\left(0 \leq q_{s} \leq 1\right)$ for worker information, data, and stimulus, respectively, and we call them information loss rate.

\subsection{Simulation Setting}

Based on the hypothetical system we defined in the above, we evaluate the influence of information loss on transient properties during recovery from node failures. There is one task and there are $M$ nodes capable of performing the task. We consider the loss rate is identical among information, that is, $q_{w}=q_{s}=q_{d}=$ $q$. There is no delay in communication. Parameters are summarized in Table 1. 


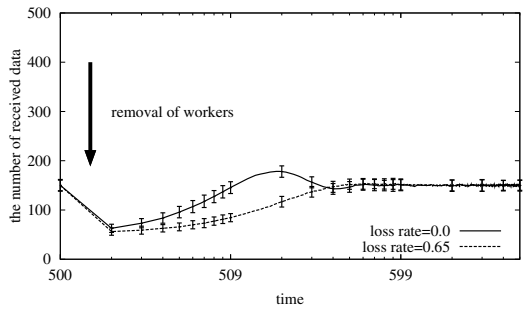

(a) The number of received data

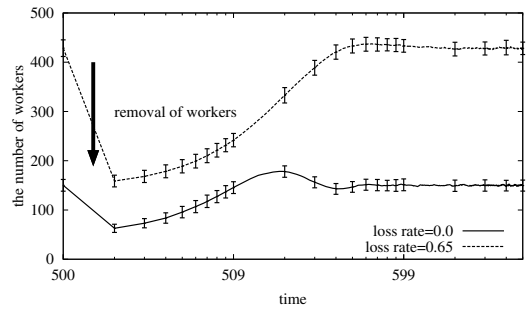

(b) The number of workers

Fig. 1. Influence of information loss during recovery from node failure

$\delta=0.15$ means that a central unit wants to receive as many as 150 data from nodes every $I_{d}$ seconds. Each simulation run lasts for 1,000 seconds. The fraction $r_{f}\left(0 \leq r_{f} \leq 1\right)$ of workers, i.e. nodes with $X_{i}(k)=1$, are randomly selected and removed at 500.5 seconds, but $M$ is unchanged reflecting that a central unit is not aware of node removal. In the following, we show averages of 100 simulation runs.

\subsection{Simulation Evaluation}

Figure 1 shows temporal changes of the number of data received by a central unit and the number of workers in the network for the information loss rates of 0.0 and 0.65 . We show averages and standard deviation. As shown in Fig. 1(a) independently of the information loss rate, a central unit eventually resumes receiving the desired number of data after node failures. Regarding the transient behavior, the number of received data for higher loss rate slowly increases, while one for no loss increases faster and even overshoots. In this simulation model, the loss of stimulus disturbs appropriate state setting of nodes and as a result higher loss rate leads to slower dynamics. On the contrary, when there is no loss of information, a node can receive a stimulus and change its state appropriately. As a result, the number of workers rapidly increases. However, because of hysteresis in the stimulus calculation, it once overshoots and then reaches the equilibrium.

Figure 1(b) shows the number of workers is larger with the larger information loss rate. When worker information is frequently lost, the stimulus becomes larger than the case without information loss to maintain the underestimated number of workers around 150. Since the loss rate of data is identical to that of worker information, the number of data that a central unit is kept around 150 as show in Fig. 1(a).

Next we evaluate the recovery time $T_{r}$, which is defined as time required for the number of received data to continuously exceed $96 \%$ of the targeted value, which is derived as $\delta \cdot M$, after node failures. In Fig. 2, we depict $T_{r}$ against the information loss rate $q$ from 0.0 to 0.5 and the node failure rate $r_{f}$ from 0.0 to 


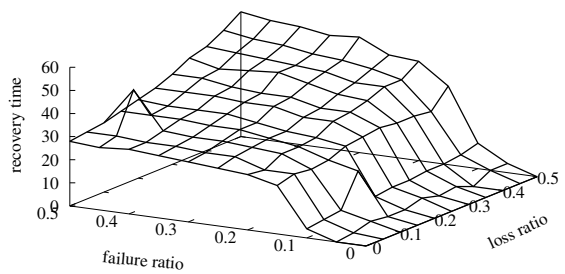

Fig. 2. Influence of information loss on the recovery time

0.5. As shown in Fig. 2, the recovery time increases as the rate of information loss increases. For example, the recovery time for $q=0.0$ and $r_{f}=0.5$ is 28 seconds. When the information loss rate is 0.5 , the recovery time becomes 54 seconds. Therefore, the increase is as much as double. In the next section, through mathematical analysis, we investigate the influence of control parameters on the recovery time to have faster convergence.

\section{Mathematical Analysis of Response Threshold Model}

From simulation-based analysis, it is shown that the number of workers increases as the loss rate becomes higher. To clarify the range of the loss rate for the response threshold model to be effective, we derive existence conditions of an equilibrium state. We also formulate the recovery time and clarify parameters important to shorten the recovery time.

\subsection{Analytical Model Considering Information Loss}

As explained in section 2, the number of workers in a system returns to the target value after removal of workers by involving non-workers with the increased stimulus. However Fig. 1 shows that there are two types of transient behavior. When a system overreacts to the decrease in the number of workers, it often results in overshooting and further leads to oscillation as illustrated in the left of Fig. 3 Even in that case, it eventually approaches to the target value or the equilibrium state as shown in the left figure. On the contrary, with moderate adaptation, the number of workers steadily increases toward the desired level without oscillation, while it would take longer than the case of aggressive adaptation as shown in the right of Fig. 3 .

An information network is a discrete system, where a node intermittently emits and receives a chunk of data called packet and performs predetermined algorithms. However in this paper, for simplicity of analysis, we adopt continuoustime modeling and analyze the effect of information loss on transient behavior, i.e. magnitude of oscillation during recovery from node failure and recovery time 

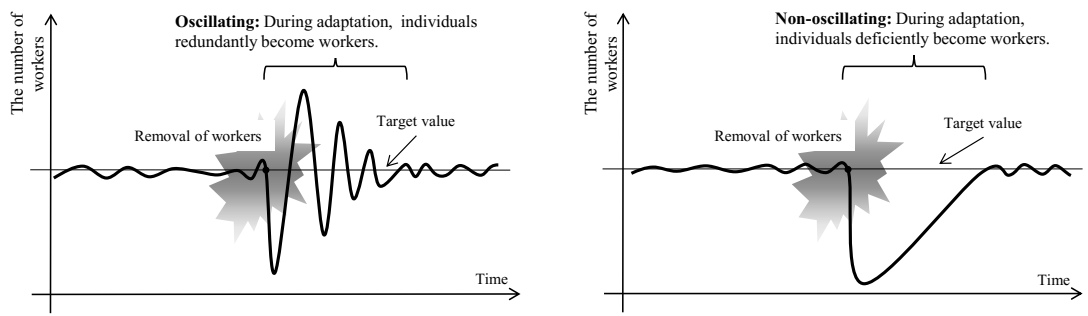

Fig. 3. Transient behavior with/without oscillation

of the response threshold model. The validity of analysis will be verified through comparison with simulation results.

Considering loss and reception of information as Bernoulli trials, the expected number of workers and non-workers which receive the stimulus information are formulated as $\left(1-q_{s}\right) n_{w}$ and $\left(1-q_{s}\right)\left(M-D-n_{w}\right)$, respectively. Here, variable $n_{w}$ is the expected number of workers in a colony. Constant $D(0 \leq D \leq M)$ is the number of dead workers, assuming that a set of individuals are statically removed from a colony. Then, the expected number $n_{s}$ of the worker information that a central unit receives from workers is formulated as $\left(1-q_{w}\right) n_{w}$. Consequently, the temporal dynamics of the expected value $s$ of the stimulus intensity and the expected number $n_{w}$ of workers can be formulated as follows.

$$
\left[\begin{array}{c}
\frac{d s}{d t} \\
\frac{d n_{w}}{d t}
\end{array}\right]=\left[\begin{array}{l}
\delta-\frac{\left(1-q_{w}\right) n_{s}}{M} \\
-p\left(1-q_{s}\right) n_{w}+\frac{s^{2}}{s^{2}+\theta^{2}}\left(1-q_{s}\right)\left(M-D-n_{w}\right)
\end{array}\right]
$$

Here we assume that threshold $\theta$ is identical among individuals.

\subsection{Characteristic Analysis of Equilibrium State}

From Eq. (44), we derive the equilibrium state $\left[\bar{s} \bar{n}_{w}\right]^{T}$, where the time variation of the expected values $s$ and $n_{w}$ are zero.

$$
\left[\begin{array}{c}
\bar{s} \\
\bar{n}_{w}
\end{array}\right]=\left[\begin{array}{l}
\theta \sqrt{\frac{p \delta}{\left(1-\frac{D}{M}\right)\left(1-q_{w}\right)-\delta(1+p)}} \\
\frac{\delta M}{1-q_{w}}
\end{array}\right]
$$

Since the stimulus intensity is a positive real number, $(1-D / M)\left(1-q_{w}\right)-\delta(1+$ $p)>0$ must hold. At the same time, $\delta M /\left(1-q_{w}\right)<M-D$ must hold so that the number of workers is smaller than the population. Therefore, the condition that a feasible equilibrium state exists is given by the following inequality.

$$
(1-f)\left(1-q_{w}\right)-\delta(1+p)>0
$$

$f$ is $D / M$, that is the ratio of the number of dead individuals to the original colony size. 


\subsection{Characteristic Analysis of Transient State}

Based on the linear stability theory, transient behavior of a linear system represented as $d \mathbf{x} / d t=A \mathbf{x}$ can be analyzed by evaluating eigenvalues of matrix $A$. An eigenvalue $\lambda_{i}$ is generally formulated as $\alpha_{i}+j \beta_{i}$, where $j$ is imaginary $\sqrt{-1}$ and $\alpha_{i}$ and $\beta_{i}$ are real numbers. $x_{i}(k)$ is mapped onto $z_{i}(k)=z_{i}(0) \exp ^{\left(\alpha_{i}+j \beta_{i}\right) k}=$ $z_{i}(0) \exp ^{\alpha_{i} k}\left(\cos \beta_{i} k+j \sin \beta_{i} k\right)$ by linearly converting $\mathbf{x}$. Therefore, the dynamics with $\forall_{i} \alpha_{i}<0$ has asymptotic stability, and smaller $\alpha_{i}$ leads to longer time for $d z_{i} / d t$ to converge to 0 . In addition, the dynamics with $\forall_{i} \beta_{i}=0$ is stable. $\alpha_{i}$ is specifically called damping factor in this paper.

Linearizing the nonlinear analytical model defined by Eq. (4), we can analyze influence of information loss on transient behavior during recovery from node failures. Firstly, we derive the Jacobian matrix from the nonlinear analytical model. We define $r$ as the fraction of workers in a colony, i.e. $r=n_{w} / M$. Then, dynamics $d s / d t$ and $d r / d t$ can be formulated as follows.

$$
\left[\begin{array}{l}
\frac{d s}{d t} \\
\frac{d r}{d t}
\end{array}\right]=\left[\begin{array}{l}
\delta-\left(1-q_{w}\right) r \\
-p\left(1-q_{s}\right) r+\frac{s^{2}}{s^{2}+\theta^{2}}\left(1-q_{s}\right)(1-f-r)
\end{array}\right]
$$

To adopt the linear stability theory, we linearize Eq. (7) at the equilibrium state $[\bar{s} \bar{r}]^{T}=\left[\theta \sqrt{\frac{p \delta}{(1-f)\left(1-q_{w}\right)-\delta(1+p)}} \frac{\delta}{1-q_{w}}\right]^{T}$ by Taylor expansion. Then, the dynamics of error $\mathbf{e}=\left[\begin{array}{ll}e_{s} & e_{r}\end{array}\right]^{T}$ between the equilibrium state $[\bar{s} \bar{r}]^{T}$ and the state $[s r]^{T}$, i.e. $\mathbf{e}=\left[\begin{array}{ll}s & r\end{array}\right]^{T}-[\bar{s} \bar{r}]^{T}$, can be formulated as the basic linear equation $d \mathbf{e} / d t=A \mathbf{e}$ as follows, where $A$ is the Jacobian matrix.

$$
\left[\begin{array}{c}
\frac{d e_{s}}{d t} \\
\frac{d e_{r}}{d t}
\end{array}\right]=\left[\begin{array}{cc}
0 & -\left(1-q_{w}\right) \\
\left(1-q_{s}\right)(1-f-\bar{r}) \frac{2 \bar{s} \theta^{2}}{\left(\bar{s}^{2}+\theta^{2}\right)^{2}} & -\left(1-q_{s}\right)\left(p+\frac{\bar{s}^{2}}{\bar{s}^{2}+\theta^{2}}\right)
\end{array}\right]\left[\begin{array}{l}
e_{s} \\
e_{r}
\end{array}\right]
$$

Next, we derive eigenvalues of the matrix $A$ from a characteristic equation $\operatorname{det}|A-\lambda I|=0$. Since the matrix $A$ in Eq. (8) is in the form of $[0 a ; b c]$, an eigenvalue is formulated as $0.5\left(c \pm \sqrt{c^{2}+4 a b}\right)$. Specifically, $c$ and $a b$ are formulated as follows, by substituting $\bar{s}$ of Eq. (5).

$$
\left\{\begin{array}{l}
c=-\left(1-q_{s}\right) p\left\{1+\frac{\delta}{(1-f)\left(1-q_{w}\right)-\delta}\right\} \\
a b=-\frac{2}{\theta} \sqrt{p \delta}\left(1-q_{s}\right) \frac{\left\{(1-f)\left(1-q_{w}\right)-\delta(1+p)\right\}^{1.5}}{(1-f)\left(1-q_{w}\right)-\delta}
\end{array}\right.
$$

$c<0$ and $a b<0$ must hold for its real part to be always less than 0 . When Eq. (6) is satisfied and the equilibrium state exists, $0<1-q_{s}, 0<p$, and $\delta<\delta(1+p)<(1-f)\left(1-q_{w}\right)$ hold. Therefore, the first condition $c<0$ is always met. Similarly, because of $0<\left(1-q_{w}\right), 0<\left(1-q_{s}\right), 0<\frac{2 \bar{s} \theta^{2}}{\left(\bar{s}^{2}+\theta^{2}\right)^{2}}$, and $0<\bar{r}(1+p)<1-f-\bar{r}$, the second condition $a b<0$ is also met. Therefore a real part of the eigenvalue is always negative and as a result the state does not diffuse out of the proximity of the equilibrium state. This means that the 


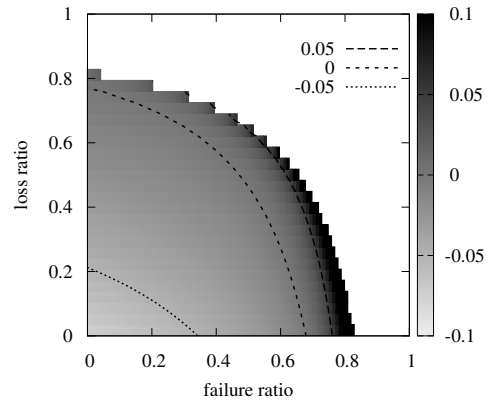

Fig. 4. Influence of loss and failure on $c^{2}+$ $4 a b$

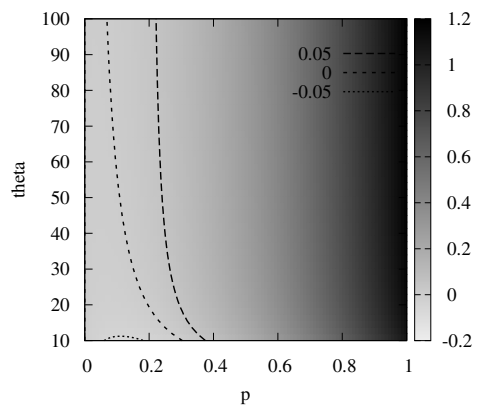

Fig. 5. Influence of parameters $p$ and $\theta$ on $c^{2}+4 a b$

response threshold model is robust against information loss once it reaches the equilibrium state.

In the following, we discuss the influence of parameters on transient behavior. For the sake of simplicity, we assume the identical loss rate, i.e. $q_{w}=q_{d}=q_{s}=q$.

Oscillating or Stable Dynamics. The dynamics of the state oscillates as shown in the left of Fig. 3 when eigenvalues of the state transition matrix have an imaginary part. In other words, a system reaches the equilibrium state without oscillation when $c^{2}+4 a b$ is positive (Fig. 3 right). On the contrary, a system wanders toward the equilibrium state when $c^{2}+4 a b$ is negative (Fig. 3 left).

Figure 4 illustrates a value of $c^{2}+4 a b$ as a function of the information loss rate and the failure rate. Parameters are set as $p=0.1, \theta=10$, and $\delta=0.15$. In the figure, the equilibrium state does not exist in the white area derived from Eq. (6) and we show contour lines of $c^{2}+4 a b=0.05,0$, and -0.05. A point with a lighter color has a smaller $c^{2}+4 a b$. The figure indicates the range that network control based on the response threshold model is feasible. The border can be formulated as $(1-f)\left(1-q_{w}\right)-\delta(1+p)=0$. Therefore, the range can be extended by choosing appropriate control parameters $\delta$ and $p$ depending on the operational condition expressed by $f$ and $q_{w}$. In the range, $c^{2}+4 a b$ becomes positive and a system steadily moves to the equilibrium when both of the information loss rate and the failure rate are large. However, $c^{2}+4 a b$ is negative in the most part. It means that the transient dynamics toward the equilibrium state basically oscillates. The oscillating dynamics causes the overshoot and redundant numbers of nodes temporarily become workers. Whereas a system finally reaches the equilibrium, it becomes a problem in a resource-limited network.

Figure 5 illustrates the influence of tunable parameters, i.e. $p$ and $\theta$ on a value of $c^{2}+4 a b$ when information loss rate $q$, failure rate $r_{f}$, increase rate $\delta$ are set at $0.1,0.1$, and 0.15 , respectively. From the figure, we can find that threshold $\theta$ has a small influence on $c^{2}+4 a b$. On the contrary, a larger $p$ can make $c^{2}+4 a b$ 


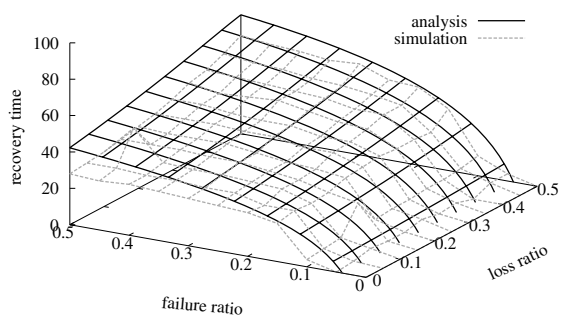

Fig. 6. Comparison of analytical simulation results

positive leading to the stable dynamics. However, a larger $p$ makes the second term of left side of Eq. (6) smaller. As a result, loss rate and failure rate need to be larger for the equilibrium state to exist. This implies that the robustness deteriorates as $p$ is large. When we adopt small $p$ to avoid loss of robustness, a system is more likely to oscillate in the transient behavior. However, from a viewpoint of a central unit, receiving a sufficient amount of data is helpful while it is intermittent. Suppressing the degree of oscillation and shortening the duration remain future work.

Recovery Time from Death. Recovery time from node failures is defined as necessary time for deviation $\Delta=\bar{n}_{w}-n_{w}$ to become as small as $4 \%$ of $\bar{n}_{w}$. Deviation $\Delta(0)$ soon after node failures is $r_{f} \cdot \bar{n}_{w}$. The deviation decreases as $\Delta(t)=\Delta(0) \exp ^{\alpha_{\max } t}$, where $\alpha_{\max }(<0)$ is a damping factor derived as $\max R e \frac{c \pm \sqrt{c^{2}+4 a b}}{2}$.

Solving the equation $0.04 \cdot \bar{n}_{w}=\Delta(0) \exp ^{\alpha_{\max } T_{r}}$, we can derive recovery time $T_{r}$ as follows.

$$
T_{r}=\frac{\log _{e} \frac{0.04}{r_{f}}}{\alpha_{\max }}
$$

Figure 6] shows the recovery time derived from Eq. (10) and obtained from simulation results. Although surfaces do not match well between analysis and simulation, the analytical result represents the relationship among failure rate, loss rate, and recovery time. Figure 7 illustrates dependence of the recovery time on these rates for the wider range. As shown in the figure, the maximum recovery time is only double of the minimum. This result supports the robustness of the response threshold model considering that loss often has an exponential influence on conventional control.

Finally, Fig. 8 illustrates the influence of tunable parameters, i.e. quitting probability $p$ and threshold $\theta$, on the recovery time derived from Eqs. (9) and (10), when information loss rate $q$, failure rate $r_{f}$, and increasing rate $\delta$ are set 


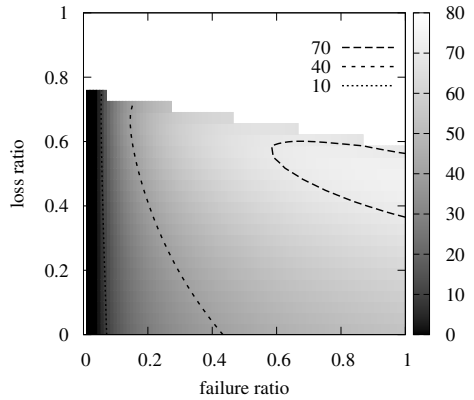

Fig. 7. Influence of loss and failure on recovery time

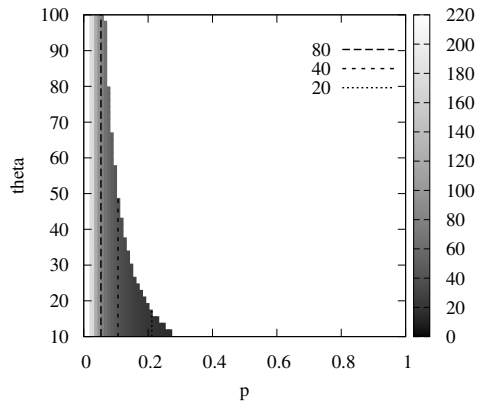

Fig. 8. Influence of parameters $p$ and $\theta$ on recovery time

at $0.0,0.5$, and 0.15 , respectively. In the white area, the equilibrium state does not exist. The figure shows that threshold $\theta$ does not have much influence on the recovery time for the fixed quitting probability $p$, but a smaller $\theta$ enables wider range of adaptation of quitting probability $p$. To make the recovery time shorter, we should have a small $\theta$ and a large $p$. When $\theta$ is 10 , the recovery time decreases from 42.3 to 21.1 by changing quitting probability from 0.1 , corresponding to the largest recovery time in Fig. 2, to 0.2 .

\section{Conclusion and Future Work}

In this paper, we investigate transient behavior during recovery from failures of individuals in the lossy environment. Results show that the response threshold model is robust against failure and loss. We further analytically clarify the influence of the environmental condition, i.e. loss rate $q$ and failure rate $r_{f}$, and the control parameters, i.e. threshold $\theta$ and quitting probability $p$, on the oscillation and the recovery time.

As future work, we plan to consider suppression of oscillation in the transient phase to have more stable and faster convergence from failures. Furthermore, we also need to investigate the distribution of the number of workers and the number of received data, because we only consider expected values and equilibrium state in the paper.

Acknowledgement. This research was supported in part by Grant-in-Aid for JSPS Fellows of Japan Society for the Promotion of Science (JSPS) and International Collaborative Research Grant of the National Institute of Information and Communications Technology, Japan. 


\section{References}

1. Bonabeau, E., Theraulaz, G., Deneubourg, J.-L., Aron, S., Camazine, S.: Selforganization in social insects. Trends in Ecology and Evolution 12, 188-193 (1997)

2. Duarte, A., Pen, I., Keller, L., Weissing, F.J.: Evolution of self-organized division of labor in a response threshold model. Behavioral Ecology and Sociobiology 66, 947-957 (2012)

3. Auchmuty, J.F.G.: Bifurcation analysis of nonlinear reaction-diffusion equations I. Evolution equations and the steady state solutions. Bullebtin of Mathematical Biology 37, 323-365 (1975)

4. Nishimura, J., Friedman, E.J.: Robust convergence in pulse-coupled oscillators with delays. Physical Review Letters 106, 194101 (2011)

5. Gutjahr, W.J.: A graph-based ant system and its convergence. Future Generation Computer Systems 16, 873-888 (2000)

6. Dressler, F., Akan, O.B.: A survey on bio-inspired networking. Computer Networks 54, 881-900 (2010)

7. Meisel, M., Pappasand, V., Zhang, L.: A taxonomy of biologically inspired research in computer networking. Computer Networks 54, 901-916 (2010)

8. Tyrrell, A., Auer, G., Bettstetter, C.: On the accuracy of firefly synchronization with delays. In: Proceedings of the International Symposium on Applied Sciences on Biomedical and Communication Technologies, pp. 1-5 (October 2008)

9. Hyodo, K., Wakamiya, N., Nakaguchi, E., Murata, M., Kubo, Y., Yanagihara, K.: Reaction-diffusion based autonomous control of wireless sensor networks. International Journal of Sensor Networks 7, 189-198 (2010)

10. Bonabeau, E., Sobkowski, A., Theraulaz, G., Deneubourg, J.L.: Adaptive task allocation inspired by a model of division of labor in social insects. In: Proceedings of the International Conference on Biocomputing and Emergent Computation, pp. 36-45 (January 1997)

11. Labella, T., Dressler, F.: A bio-inspired architecture for division of labour in SANETs. Advances in Biologically Inspired Information Systems, 211-230 (December 2007)

12. Janacik, P., Heimfarth, T., Rammig, F.: Emergent topology control based on division of labour in ants. In: Proceedings of the International Conference on Advanced Information Networking and Applications, pp. 733-740 (April 2006)

13. Bonabeau, E., Henaux, F., Guérin, S., Snyers, D., Kuntz, P., Theraulaz, G.: Routing in telecommunications networks with ant-like agents. In: Albayrak, Ş., Garijo, F.J. (eds.) IATA 1998. LNCS (LNAI), vol. 1437, pp. 60-71. Springer, Heidelberg (1998)

14. Sasabe, M., Wakamiya, N., Murata, M., Miyahara, H.: Media streaming on P2P networks with bio-inspired cache replacement algorithm. In: Proceedings of the International Workshop on Biologically Inspired Approaches to Advanced Information Technology, pp. 380-395 (May 2004) 